\title{
Endoloop versus intracorporeal knotting: Comparison of two appendiceal ligation methods during laparoscopic appendectomy
}

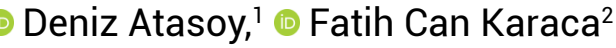 \\ 'Departmen of General Surgery, Medicana Çamlıca Hospital, İstanbul, Turkey \\ ${ }^{2}$ Departmen of General Surgery, Bilgi University School of Health Sciences, İstanbul, Turkey
}

\begin{abstract}
Introduction: The laparoscopic approach to appendectomy surgery led to various appendiceal ligation methods; however, the ideal technique for appendiceal stump closure has yet to be determined. This study is a comparison of intracorporeal knotting (IK) and Endoloop (Ethicon, Inc., Somerville, NJ, USA) (EL) techniques for appendiceal stump closure during laparoscopic appendectomy (LA) surgery.
\end{abstract}

Materials and Methods: All of the LAs included in the study were performed by only 2 surgeons between June 2013 and June 2018 and the results were retrieved retrospectively. The patients were divided into an IK group and an EL group. Early postoperative complications ( $<30$ days) were evaluated. All of the LAs were performed using 3 trocars. In the IK group, the appendiceal base was ligated with a manually constructed single intracorporeal knot. In the EL group, the appendiceal base was ligated with a single Endoloop tie (Vicryl ligature; Ethicon, Inc., Somerville, NJ, USA). A LigaSure device (Medtronic, Inc., Minneapolis, MN, USA) was utilized in both techniques for transection of the appendix. The specimen was extracted through the umbilical port within an Endobag (Medtronic, Inc., Minneapolis, MN, USA). A normal diet was administered within 6 hours postoperatively and the patients were discharged the day after the operation.

Results: The IK group consisted of 54 patients and the EL group comprised 75 patients. The groups were similar regarding gender, age, body mass index, and American Society of Anesthesiologists score. Although the operation time tended to be longer in the IK group, the difference was not statistically significant (48.46 \pm 23.85 vs. $45.36 \pm 22.28$ minutes; $p=0.459)$. In the EL group, the drain was retained in 1 patient for 2 weeks due to liver cirrhosis and 1 patient had an intraabdominal abscess. The latter patient was treated with percutaneous drainage and antibiotherapy.

Conclusion: The IK technique and the EL technique had similar results. Due to its lower cost and wider availability, IK might be suggested over the EL technique. Eliminating the dependence on commercial products and providing the means for the development of advanced laparoscopic skills are additional benefits of the IK technique.

Keywords: Appendectomy; Endoloop; intracorporeal knotting; laparoscopy. 


\section{Introduction}

Different methods exist for the ligation of the appendiceal stump during laparoscopic appendectomy (LA). These methods include stapler, metal clip, Hem-o-lok clip, endoloop (EL), and intracorporeal knotting (IK). ${ }^{[1]}$ Although there are many studies comparing these different techniques among themselves, there is no consensus on the ideal method yet.

In this study, we aimed to compare the IK and EL methods for appendiceal stump ligation during LA surgery.

\section{Materials and Methods}

Between June 2013-June 2018, all LAs performed by two surgeons were retrieved retrospectively. Only IK and EL techniques for ligation of the appendiceal stump were included to the study. Appendectomy operations performed with linear staplers were excluded. Patients were divided into two groups (IK and EL groups). Stump closure methods were chosen on the discretion of surgeon. Signed informed consent was obtained from all patients.

The patients' demographics (age, gender, BMI (body mass index), ASA score) were retrieved from patients' files. Variables including operation time, drain use, and length of hospital stay were recorded. The patients were called for a follow-up visit at 1 week postoperatively. Early (<30 days) postoperative complications were included in the study.

\section{Surgical technique}

All patients were administered intravenous 2nd generation Cephalosporin antibiotic, $30 \mathrm{~min}$ before the incision, for prophylaxis. A urinary catheter was inserted routinely and removed before awakening of the patient. The operation was performed through three ports. The pneumoperitoneum was established through inferior umbilical incision. In patients without previous abdominal operation, a Veress needle was utilized to enter the abdomen. In patients with previous abdominal operations or in pregnant patients, open Hasson technique was utilized to enter the abdomen. Intraabdominal pressure was adjusted to $12 \mathrm{mmHg}$. A $10 \mathrm{~mm}$ trocar was inserted from the umbilical incision. A 30-degree scope was inserted through this trocar. The $2^{\text {nd }}$ trocar was placed from the left lower abdominal quadrant. This trocar was either $5 \mathrm{~mm}$ or $10 \mathrm{~mm}$, depending on the surgeon's choice. The $3^{\text {rd }} 5 \mathrm{~mm}$ trocar was inserted from the suprapubic area, paying attention to the urinary bladder.
The patients were given a slight Trendelenburg position with slight left tilt. Following dissection of the mesoappendix, the base of the appendix was prepared for ligation. In patients, to whom EL (Vicryl Endoloop Ligature, J\&J medical devices) technique was performed, a single EL was placed to the appendiceal base and tied properly (Fig. 1). The appendix was transected distal to the tie with a Ligasure (LigaSure Vessel Sealing System, Valleylab, Boulder, CO).

In patients to whom IK was performed, a Vicryl (Ethicon, Johnson\&Johnson Medical devices) No: 2/0, $75 \mathrm{~cm}$ suture was utilized. Firstly, the suture was inserted through the left abdominal trocar and looped around the appendiceal base and pulled out through the same trocar. A sliding knot was made extracorporeally and pushed towards the appendiceal base with a laparoscopic dissector (Fig. 2). When the base of the appendix was reached, the knot was secured with one end of the suture in a hand extracorporeally and the other end intracorporeally with a laparoscopic dissector. The redundant ends of the suture were shortened and $2^{\text {nd }}$ and $3^{\text {rd }}$ knots were done intracorporeally. Appendiceal transection was performed distal to this suture with a Ligasure.

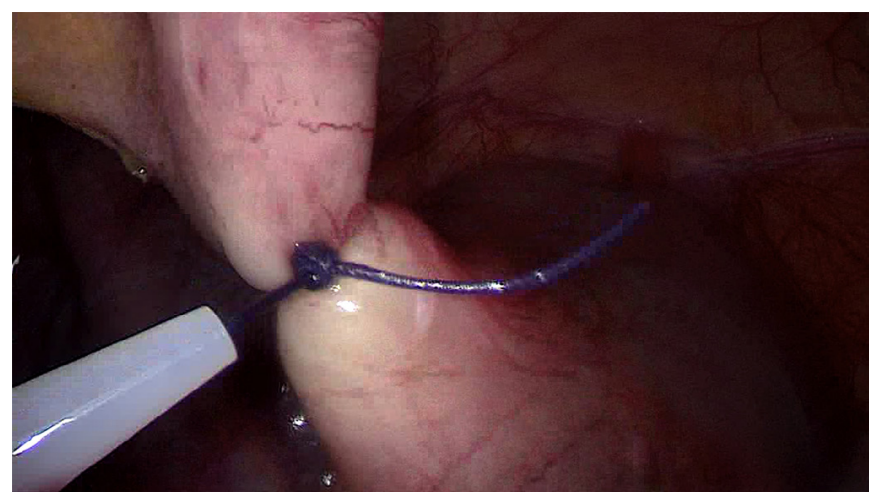

Figure 1. Ligation of the appendiceal base with endoloop.

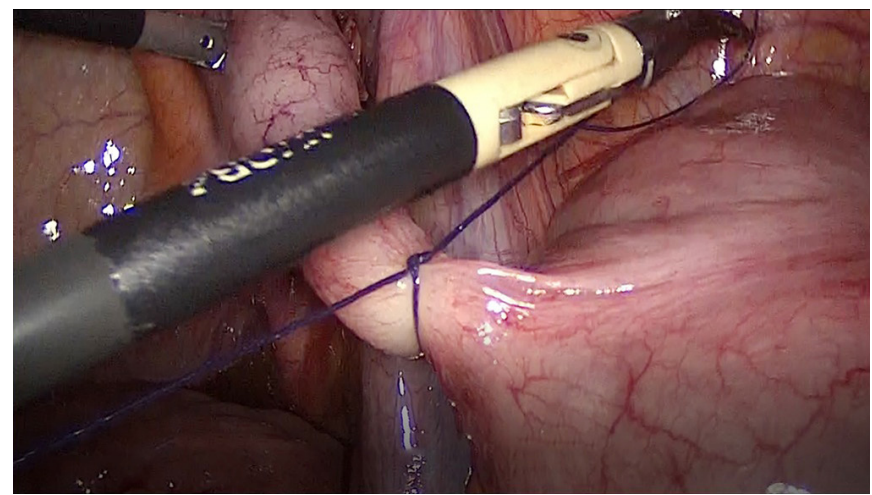

Figure 2. Ligation of the appendiceal base with intracorporeal knotting. 
Table 1. Comparisons of the intracorporeal knotting and endoloop groups

\begin{tabular}{lccc} 
& Intracorporeal knotting (IK) group & Endoloop (EL) group & p-value \\
\hline Patient number, $\mathrm{n}$ & 54 & 75 & \\
Gender, $\mathrm{n}(\%)$ & & & \\
$\quad$ Male & $27(50 \%)$ & $38(50 \%)$ & 1.000 \\
$\quad$ Female & $27(50 \%)$ & $37(50 \%)$ & 0.993 \\
Age (year) & $33.50 \pm 13.10$ & $33.48 \pm 13.17$ & 0.217 \\
BMI (kg/m ${ }^{2}$ ) & $25.96 \pm 4.19$ & $24.85 \pm 4.70$ & 0.701 \\
ASA score (median) (range, I-III) & $\mathrm{I}$ & $\mathrm{I}$ & 0.459 \\
Operation time, min & $48.46 \pm 23.85$ & $45.36 \pm 22.28$ & 1.000 \\
Drain presence, $\mathrm{n}(\%)^{\ddagger}$ & $3(5.9 \%)$ & $5(7.1 \%)$ & 0.480 \\
Length of hospital stay, day & $1.17 \pm 0.38$ & $1.23 \pm 0.53$ & 1.000 \\
Complications, $\mathrm{n}(\%) \S$ & $2(3.9 \%)$ & \\
\hline
\end{tabular}

BMI: Body Mass Index, ASA: American Society of Anesthesiologists. ${ }^{*}$ Drainage rate was calculated according to 51 patients in the IK group and 70 patients in the EL group. ${ }^{8}$ Complications were retrieved from 49 patients in the IK group and 51 patients in the EL group.

The specimen was extracted within an endobag in both of the techniques through the umbilical trocar-site. Fascial defects of $10 \mathrm{~mm}$ trocars were sutured with No:1 Vicryl (Ethicon, Johnson\&Johnson international). A drain was placed according to the intraabdominal findings and surgeon's discretion. All patients but one, had their drains removed before discharge. One patient with liver cirrhosis was discharged with his drain and had his drain removed 2 weeks postoperatively.

At postoperative $6^{\text {th }}$ hour, patients were given a normal diet and discharged the day after operation.

\section{Statistical analysis}

In the evaluation of the data, number (n), percentage, mean, and standard deviation were used as descriptive statistics. The suitability of the variables to normal distribution was evaluated by Kolmogorov Smirnov test. Student-t test or Mann-Whitney U test were used for paired group comparisons; chi-square test or Fisher exact test were used for the comparison of qualitative data. The analyzes were performed using Epi Info Version 3.5.4 and $\mathrm{p}<0.05$ was considered statistically significant.

\section{Results}

A total of 129 patients were included to the study. During the study period, two patients underwent stapler appendectomy operations. These patients had inflamed appendiceal bases, therefore the appendectomies were performed at the cecal level with linear stapler transections.
There were 65 male and 64 female patients. Comparisons of the groups were listed in Table 1 . There was no statistically significant difference between the groups regarding gender, age, BMI, and ASA score. In the IK group, there were two patients one having 20 wks. and the other having 12 wks. of pregnancy. Regarding operation times, mean durations in the IK group were slightly longer than the EL group, but the difference was not statistically significant $(48.46 \pm 23.85$ vs. $45.36 \pm 22.28, \mathrm{p}=0.459)$. Drains were placed in 3 patients in IK group and 5 patients in EL group ( $\mathrm{p}=1.000)$. Due to liver cirrhosis and ascites drainage, the drain was removed 2 wks. postoperatively in one of the patients in the EL group. Length of hospital stay was similar between the groups $(1.17 \pm 0.38$ vs. $1.23 \pm 0.53, p=0.480$ ). Considering complications, the difference between groups was not statistically significant ( $p=1.000$ ). In the EL group, on the postoperative 1st week, a collection in the appendiceal bed was seen. Percutaneous aspiration was performed and intravenous antibiotherapy was started electively. Patient recovered uneventfully, without a need for drain placement. Other complications were minor wound problems. There was no mortality.

\section{Discussion}

According to the results of this study, the IK technique was as safe and feasible as the EL technique for the ligation of the appendiceal stump. Although statistically insignificant, the operation times were 3 min longer in the IK technique than the EL technique. 
Some of the techniques for ligation of the appendiceal stump during LA include staplers, metal clips, Hem-o-lok clips $\left(\right.$ Weck $\left.^{\circledR}\right)$, EL, and IK. One of the most important factors in choosing among these methods is cost. Regarding the cost, metal clips, Hem-o-lok clips, and IK seem more advantageous over the staplers and ELs. ${ }^{[1-9]}$

In this study, due to our habit, for ligation of the appendiceal stump we used Vicryl (Ethicon ${ }^{\circledR}$ ) No: $2 / 0,75 \mathrm{~cm} \mathrm{su-}$ ture. To reduce the cost, silk sutures could also be used as reported by Bozkurt et al. ${ }^{[3]}$ In addition, to reduce the cost further, use of glove endobags for specimen retrieval were also suggested. ${ }^{[10]}$

A study similar to the present one was reported by Bali et al. $^{[4]}$ They reported statistically significantly longer operation times in the IK group than the EL group. The difference was $5 \mathrm{~min}$. There were no differences regarding complications and length of hospital stays.

The postoperative infectious complications after appendectomy were reported to be influenced by the grade of appendicitis. ${ }^{[11]}$ Based on this information, Gonenc and colleagues $^{[2]}$ compared the IK and metal clipping techniques for the ligation of the appendiceal stump, in only non-complicated appendicitis patients. Operation time in the IK group was 15 min longer than the metal clipping group with statistical significance. Complication rates and length of hospital stays were similar between the groups.

Differing from the IK in this study, some studies reported invaginating purse string suturing techniques for the appendiceal stump. ${ }^{[8,12]}$ We believe that, this method, which looks like the continuation of habits in open appendectomy technique, decreased with laparoscopic approach. Strzałka et al. ${ }^{[8]}$ compared endoscopic staplers, titanium clips, and invaginating suture techniques and reported that invaginating sutures resulted with longer length of hospital stays, longer operation times, and higher complication rates compared to other methods.

Delibegovic and colleagues compared Hem-o-lok and EL techniques. ${ }^{[5]}$ They utilized two clips or two ELs for the ligation of the appendiceal stump. In our technique, we utilized a single EL or IK for appendiceal ligation. In daily practice, techniques differ depending on the surgical schools. As well as distal to double sutures, some appendix transections are performed distal to a single suture or a single clip. Similarly, together with appendiceal transections between clips, like in this study, transections with Ligasure are also widely used. Comparisons of these different methods may be the subject of further studies.

In Hem-o-lok technique, the length of the clip limits the maximal diameter of an appendiceal stump to be clipped.

${ }^{[8]}$ In wider appendiceal base diameters, application of this clip is limited. To reduce the diameter, firstly application of an intracorporeal suture, then application of a Hem-o-lok clip was described. ${ }^{[12]}$ Similarly, appendiceal diameter could also cause some difficulties during metal clipping. Alis et al. ${ }^{[1]}$ described 'cross-clipping' method in cases where the appendiceal base is larger than the clip size. In another study, special titanium clips allowed ligation of appendix bases of up to $20 \mathrm{~mm}$ in diameter, however for the application of these clips, trocars with diameter of 12.5-13 mm were needed..$^{[8]}$ Likewise, same diameter of trocars is also needed for stapler application and this condition poses disadvantage against $10-11 \mathrm{~mm}$ trocars needed for Hem-o-lok clip or titanium clip applications. Wilson et al., ${ }^{[13]}$ compared EL and Hem-o-lok clipping techniques in LA, and reported that Hem-o-lok clipping had shorter operation times and lower cost.

In a randomized study, Hem-o-lok clipping and clipping with absorbable polymer clips (Lapro-clip [Covidien, NC, USA]) were reported to have similar complication rates. ${ }^{[14]}$ In that study, stumps were ligated with one or two clips, depending on the surgeon's discretion. There were no differences among patients with one or two clips. The polymer clips used in that study were reported to be absorbed in 90 days, however were more expensive than Hem-o-lok clips.

Shadhu et al. ${ }^{[12]}$ reported that, in the IK method, patients were hospitalized for a longer period of time and had higher rates of drainage. The reason was attributed to the low confidence of surgeons about the safety of the knotting.

It could be assumed that, IK application is proportional to the surgeon's experience. From that point of view, IK technique may provide medium to increase surgeons' skills. ${ }^{[2,4]}$ Gonenc et al., ${ }^{[2]}$ suggested experienced surgeons to accompany the inexperienced surgeons during their first 10-15 LAs.

One of the most important disadvantages of the IK technique may be the difficulty of application compared to other techniques. This technique requires laparoscopic knotting skills and experience in feeling the tension of the knots on the tissues. 
One of the factors that is effective in the preference of ligation of the appendiceal stump is the ease of application. Techniques that can be applied automatically can therefore be preferred over manual techniques.

Additional point of attention in EL method is that the EL knot can be stacked to the surrounding small intestinal serosa and meso during application. In this case, it is not possible to loosen the knot of the EL, so additional ELs may be needed. However, in the IK method, it is possible to loosen the knot and remove the intervening tissues and tighten the knot again.

The ideal appendix stump closure during LA surgery has not yet been described. From an ideal appendix stump closure method, it can be expected to have the least possible complication rates, should be easily accessible, inexpensive and easily applicable.

One of the limitations of our study was its retrospective nature. Therefore, some data loss and patient losses in the postoperative follow-up cannot be prevented. A similar study with prospective randomized design may provide further information.

According to the results of this study, the IK method can be recommended instead of the EL method, due to its low cost and ease of accessibility. Another advantage of the IK method is that it eliminates dependence on commercial products such as EL. In addition, the IK method may be useful in preparing surgeons for advanced laparoscopic procedures.

\section{Disclosures}

Financial disclosure: There are no financial supports.

Peer-review: Externally peer-reviewed.

Conflict of Interest: None declared.

\section{References}

1. Alis H, Gonenc M, Deniztas C, Kapan S, Turhan AN. Metal endoclips for the closure of the appendiceal stump in laparoscopic appendectomy. Tech Coloproctol 2012;16:139-41.

2. Gonenc M, Gemici E, Kalayci MU, Karabulut M, Turhan AN, Alis $\mathrm{H}$. Intracorporeal knotting versus metal endoclip application for the closure of the appendiceal stump during laparoscopic appendectomy in uncomplicated appendicitis. J
Laparoendosc Adv Surg Tech A 2012;22:231-5.[CrossRef]

3. Bozkurt MA, Unsal MG, Kapan S, Gonenc M, Dogan M, Kalayci $\mathrm{MU}$, et al. Is laparoscopic appendectomy going to be standard procedure for acute appendicitis; a 5-year single center experience with 1,788 patients. Eur J Trauma Emerg Surg 2015;41:87-9. [CrossRef]

4. Bali İ, Karateke F, Özyazıcı S, Kuvvetli A, Oruç C, Menekşe E, et al. Comparison of intracorporeal knotting and endoloop for stump closure in laparoscopic appendectomy. Ulus Travma Acil Cerrahi Derg 2015;21:446-9. [CrossRef]

5. Delibegović S, Matović E. Hem-o-lok plastic clips in securing of the base of the appendix during laparoscopic appendectomy. Surg Endosc 2009;23:2851-4. [CrossRef]

6. Wilson M, Maniam P, Ibrahim A, Makaram N, Knight SR, Patil $P$. Polymeric clips are a quicker and cheaper alternative to endoscopic ligatures for securing the appendiceal stump during laparoscopic appendicectomy. Ann R Coll Surg Engl 2018;100:454-8. [CrossRef]

7. Reinke CE, Tabone LE, Fong P, Yoo JS, Park CW. Safety and Efficacy of Polymeric Clips for Appendiceal Stump Closure. JSLS 2016;20. [CrossRef]

8. Strzałka M, Matyja M, Rembiasz K. Comparison of the results of laparoscopic appendectomies with application of different techniques for closure of the appendicular stump. World $\mathrm{J}$ Emerg Surg 2016;11:4. [CrossRef]

9. Rickert A, Krüger CM, Runkel N, Kuthe A, Köninger J, JansenWinkeln B, et al. The TICAP-Study (titanium clips for appendicular stump closure): A prospective multicentre observational study on appendicular stump closure with an innovative titanium clip. BMC Surg 2015;15:85. [CrossRef]

10. Aziret $M$, Çetinkünar $S$, Erdem $H$, Kahramanca Ş, Bozkurt $H$, Dülgeroğlu $\mathrm{O}$, et al. Comparison of open appendectomy and laparoscopic appendectomy with laparoscopic intracorporeal knotting and glove endobag techniques: A prospective observational study. Turk J Surg 2017;33:258-66. [CrossRef]

11. Malagon AM, Arteaga-Gonzalez I, Rodriguez-Ballester L. Outcomes after laparoscopic treatment of complicated versus uncomplicated acute appendicitis: a prospective, comparative trial. J Laparoendosc Adv Surg Tech A 2009;19:721-5.

12. Shadhu $K$, Ramlagun $D$, Wang $Y$, Ping $X$, Chen $T$, Zhu $Y$, et al. Re-evaluation of purse string suture in laparoscopic appendectomy. Surg Endosc 2019. [CrossRef]

13. Wilson M, Maniam P, Ibrahim A, Makaram N, Knight SR, Patil P. Polymeric clips are a quicker and cheaper alternative to endoscopic ligatures for securing the appendiceal stump during laparoscopic appendicectomy. Ann R Coll Surg Engl 2018;100:454-8. [CrossRef]

14. Lv B, Zhang $X$, Li J, Leng S, Li S, Zeng Y, et al. Absorbable polymeric surgical clips for appendicular stump closure: A randomized control trial of laparoscopic appendectomy with lapro-clips. Oncotarget 2016;7:41265-73. [CrossRef] 